\title{
Astrochemistry Results from the Spitzer c2d Project
}

\author{
Neal J. Evans II $^{1}$ and the c2d Team ${ }^{2}$ \\ ${ }^{1}$ Department of Astronomy, The University of Texas at Austin, Austin, TX 78712, USA \\ email: nje@astro.as.utexas.edu \\ ${ }^{2}$ http://peggysue.as.utexas.edu/SIRTF/
}

\begin{abstract}
The early results on astrochemistry from the Cores to Disks (c2d) Legacy program are summarized. The $\mathrm{c} 2 \mathrm{~d}$ program focuses on the formation of low-mass stars in nearby (within about $300 \mathrm{pc}$ ) clouds. Spectroscopy with IRS includes the following topics: ices seen against background stars, in protostellar envelopes, and in disks; grain growth and mineralogy, PAHs, and gas in circumstellar disks.
\end{abstract}

Keywords. dust — infrared: ISM — protoplanetary disks

\section{Introduction}

The c2d Legacy Program (Evans et al. 2003) is using the instruments of the Spitzer Space Telescope to trace the evolution of molecular material from the prestellar, dense core stage through the formation of planet-forming disks. Five large clouds (covering a total of $\sim 20$ sq. deg.) and about 90 smaller cores are being imaged with both IRAC (4 bands from 3.6 to $8 \mu \mathrm{m}$ ) and MIPS (3 bands from 70 to $160 \mu \mathrm{m}$ ). To study the timescale for disk dissipation, we are obtaining photometry on $\sim 190$ weak-line T Tauri stars. The evolution of the physical and chemical state of the matter is being studied with spectroscopy of over 170 targets (down to sub-solar mass stars, as faint as a few mJy) with IRS. Finally, a large data base of complementary observations, from optical to millimeter wavelengths, is being assembled. Because the IRS observations bear most directly on astrochemistry, I will focus on them here.

\section{Evolution of Ices}

Spectroscopy of background stars can reveal the nature of the dust and ice in dense regions before any star has formed. The sensitivity of IRS on Spitzer allows study of lines of sight with very high extinction. Bergin et al. (2005) and Knez et al. (2005) have found large abundances of $\mathrm{CO}_{2}$ ice, relative to $\mathrm{H}_{2} \mathrm{O}$ ice, toward highly extincted background stars. The shape of the feature shows that the $\mathrm{CO}_{2}$ is not crystallized, indicating its pristine nature. In addition, Knez et al. (2005) detect for the first time the $6.85 \mu \mathrm{m}$ band toward background stars. Its strength is comparable to that previously observed toward embedded protostars, and thus its carrier $\left(\mathrm{NH}_{4}^{+}\right.$, tentatively) must have been formed early in the cloud history. Other, weaker features suggest the presence of $\mathrm{HCOOH}$ and $\mathrm{NH}_{3}$, but these lie on the edge of stronger features and need confirmation.

Similar spectra of embedded protostars show features of solid $\mathrm{CH}_{4}, \mathrm{CH}_{3} \mathrm{OH}, \mathrm{CO}$, $\mathrm{CO}_{2}, \mathrm{H}_{2} \mathrm{O}, \mathrm{NH}_{4}^{+}$, and $\mathrm{OCN}^{-}$(Boogert et al. 2004). Systematic trends in the shape of the $\mathrm{CO}$ and $\mathrm{CO}_{2}$ bands indicative of increased temperatures leading to ice sublimation and changing ice structure can be seen in a range of objects, similar to what was seen toward more massive and luminous objects with ISO ( $c f$. van Dishoeck 2004). 


\section{Evolution of Disks}

Work with ISO observations also showed that disks around intermediate mass stars can have a range of profiles, ranging from flared, to self-shadowed, to disks with inner holes (van Dishoeck 2004). A similar range of SEDs is seen toward lower mass stars with Spitzer. Analysis of the silicate emission features at 10 and $20 \mu \mathrm{m}$ shows evidence that the small end of the grain size distribution is increasing rapidly in disks (Kessler-Silacci et al. 2005). Considering models of rapid grain growth and settling, these observations may eventually shed light on processes such as vertical mixing and grain fragmentation.

PAH emission is detected toward at least $8 \%$ of the T Tauri stars, down to spectral type G8. The 11.3 micron feature strength suggests for some sources the presence of additional UV radiation beyond that provided by the star itself, perhaps associated with active accretion (Geers et al., in preparation). Ionized PAHs around these cool stars could also be excited by visible or near-IR photons (Mattioda et al. 2005).

With special orientations, the disk can be probed by absorption spectroscopy. Ices in such a "grazing-incidence" disk were studied by Pontoppidan et al. (2005). Because the IRS instrument is not optimized for gas-phase features, the most surprising result so far is the discovery of gas-phase absorption bands of $\mathrm{CO}_{2}, \mathrm{HCN}$, and $\mathrm{C}_{2} \mathrm{H}_{2}$ toward one young star+disk, IRS46 in Ophiuchus (Lahuis et al. 2005). Large abundances of hot, dense, chemically-rich gas coming from a region less than about $10 \mathrm{AU}$ in size suggest absorption in the inner regions of a disk. However, follow-up observations with high spectral resolution indicate a $25 \mathrm{~km} \mathrm{~s}^{-1}$ blueshift relative to the cloud velocity, raising the possibility of the footprint of a disk wind fortuitously located along the line of sight. Further observations are needed to decide between these two possible interpretations.

\section{Acknowledgements}

Support for this work, part of the Spitzer Legacy Science Program, was provided by NASA through contracts 1224608, 1230779, and 1256316 issued by the Jet Propulsion Laboratory, California Institute of Technology, under NASA contract 1407. Astrochemistry in Leiden is supported by a NWO Spinoza grant and a NOVA grant.

\section{References}

Bergin, E.A., Melnick, G.J., Gerakines, P.A., Neufeld, D.A., \& Whittet, D.C.B. 2005, Ap. J. 627, L33

Boogert, A.C.A., et al. 2004, Ap. J. Suppl 154, 359

Evans, N.J., et al. 2003, PASP 115, 965

Kessler-Silacci, J., Augereau, J.C., Dullemond, C.P., Geers, V., Lahuis, F., Evans, N.J., II, van Dishoeck, E.F., Blake, G.A., Boogert, A.C.A., Brown, J., Jørgensen, J.,K., Knez, C., \& Pontoppidan, K.M. 2005, Ap. J., in press

Knez, C., Boogert, A.C.A., Pontoppidan, K.M., Kessler-Silacci, J., Evans, N.J., II, van Dishoeck, E.F., Augereau, J.C., Blake, G.A., \& Lahuis, F. 2005, Ap. J., in press

Lahuis, F., Boogert, A.C. A., van Dishoeck, E.F., Pontoppidan, K.M., Blake, G.A., Dullemond, C.P., Evans, N.J., II, Hogerheijde, M.R., Jørgensen, J.K., Kessler-Silacci, J., \& Knez, C. 2005, Ap. J., submitted

Mattioda, A.L., Allamandola, L.J., \& Hudgins, D.M. 2005, Ap. J. 629, 1183

Pontoppidan, K.M., Dullemond, C.P., van Dishoeck, E.F., Blake, G.A., Boogert, A.C.A., Evans, N.J., Kessler-Silacci, J.E., \& Lahuis, F. 2005, Ap. J. 622, 463

van Dishoeck, E.F. 2004, ARAA 42, 119 